\title{
Results and Experiences from the Application of Digital Image Correlation in Operational Modal Analysis
}

\section{František Trebuňa, Róbert Huňady, Zdenko Bobovský, Martin Hagara}

Technical University of Kosice, Faculty of Mechanical Engineering, Department of Applied Mechanics and Mechatronics, Letná 9, 04200 Košice, Slovakia frantisek.trebuna@tuke.sk, robert.hunady@tuke.sk, zdenko.bobovsky@tuke.sk, martin.hagara@tuke.sk

\footnotetext{
Abstract: Digital Image Correlation is a modern full-field optical method that is being used for measurement of $3 D$ displacements and surface strains. The correlation systems using the high-speed digital cameras also allow perform dynamic measurements and vibration analysis. The paper deals with possibilities of using the high-speed correlation system Dantec Dynamics Q-450 for determination of modal parameters. Since, the correlation system does not have software tools for the evaluation of such type of measurement, the using of another post-processing application is necessary. The paper describes a conception and the basic functions of the software tool that is being developed for that purpose. The main attention of the paper is focused on Operational Modal Analysis.
}

Keywords: digital image correlation; Dantec Dynamics Q-450; operational modal analysis; Modan 3D

\section{Introduction}

Every structure has a tendency to vibrate under certain frequencies. This phenomenon is known as resonance. In the resonance state amplitudes can reach critical values. From this point of view, vibrations which occur in the majority of mechanical systems are undesirable not only because of the increased noise and dynamical stresses that can in some cases lead to malfunction or failure of a structure, but also due to loss of energy and decrease in efficiency. There are many cases known where excessive vibrations of one or several parts of the structure led to their damage or decreasing their lifetime. That is the reason why the dynamic analysis of a structure is part of its design process. After its completion and when putting the structure into operation, it is necessary to experimentally verify the assumptions of the analytical solution. 
Experimental estimation of modal parameters from the measured data is known as modal testing or modal analysis. We distinguish two basic types. The first type, Experimental Modal Analysis (EMA), lies in the investigation of the relation between excitation and response of the object in the frequency domain. The second type, Operational Modal Analysis (OMA), allows determine modal parameters by only measuring the response of the structure without using an artificial excitation.

Sensors of acceleration are most often used for the measurement in the experimental testing. Thanks to progress achieved in the area of computer technology, modern optical systems which are becoming more common and increasingly available allow to provide high-speed contactless and blanket measurement of space displacements or velocities of an object. This category of measurement systems includes e.g. laser vibroscanners or digital image correlation.

\section{Digital Image Correlation Method}

Digital image correlation is an optical method based on correlation principle of a random black and white pattern created on an investigated object, which is sampled by CCD cameras during its loading. This pattern imitates the object contour, deforms and moves with it together. A comparison of acquired digital images, also called correlation, is performed gradually on small image elements called facets (Fig. 1). Minimal facet size is determined by a size of created pattern in such a way that every one facet has to contain white and black color in order to ensure proper correlation. Shape of these image elements use to be squared with usual size from $15 \times 15$ to $30 \times 30$ pixels [1].

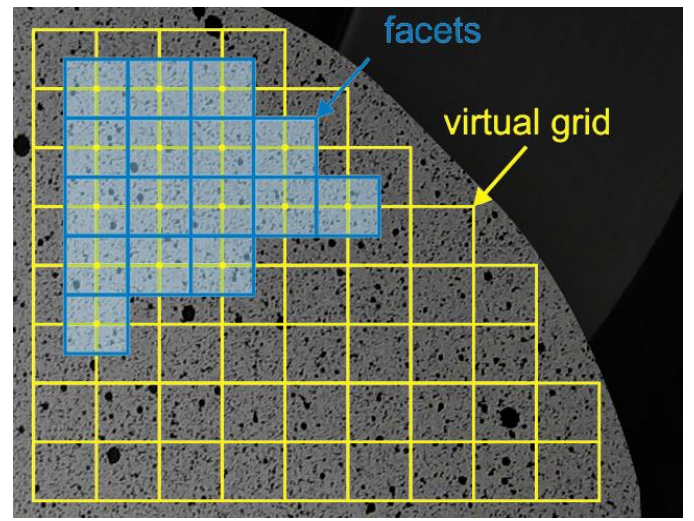

Figure 1

Virtual grid and facets on an object surface with created random black and white pattern 
There are various ways to create finer or coarser pattern (using of a spray gun, an easy coating of Xerox toner on wet white surface, a manual painting by indelible ink felt pen or a chemical etching of metal materials), but the easiest and most popular as well is a creation of pattern by white and black spray color [1].

If the sampling process is performed by one CCD camera then the correlation is constrained only for planar objects situated parallel to camera image plane. For this instance it is not possible to perform spatial analysis but only a planar one (Fig. 2).

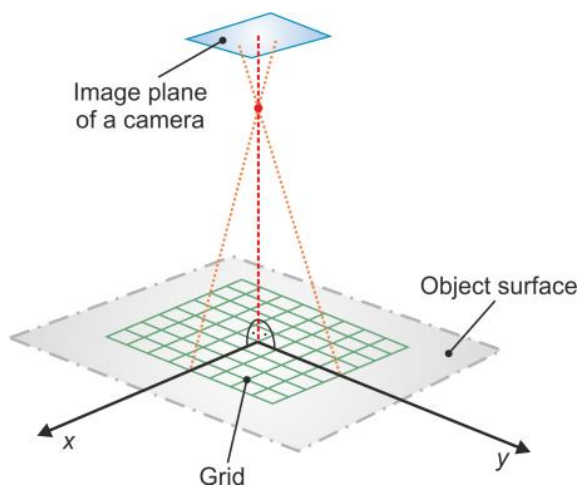

Figure 2

Principle of 2D Digital Image Correlation with one CCD camera

By a spatial analysis minimally a stereoscopic configuration of sensors is necessary. Providing that the object is observed from two different directions, the position of each object point is focused on a specific pixel of corresponding camera image plane (Fig. 3) [2]. If the cameras position, lens magnifications and all image parameters are known, it is possible to calculate the absolute threedimensional coordinates of each object point and thus make its spatial contour [3].

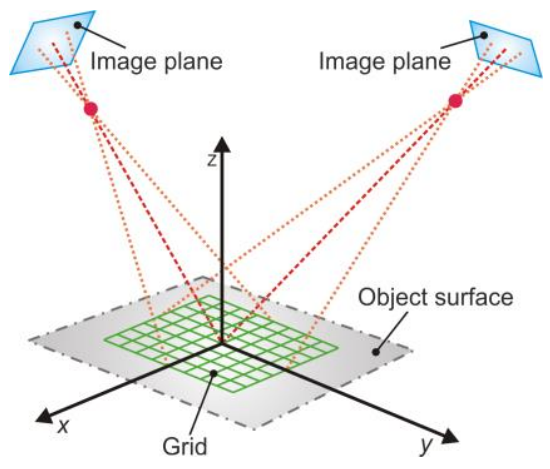

Figure 3

Principle of 3D Digital Image Correlation with two CCD cameras 
Dantec Dynamics correlation systems realize calculations with the assistance of correlation algorithm based on pseudo-affine coordinates transformation of object points from one picture to the second one. If $a_{0}, a_{1}, \ldots, a_{7}$ are the transformation parameters of potential translation, stretch, shear and distortion (Fig. 4), the coordinate transformations can be expressed $[4,5]$ :

$$
\begin{aligned}
& x_{t}\left(a_{0}, a_{1}, a_{2}, a_{3}, x, y\right)=a_{0}+a_{1} x+a_{2} y+a_{3} x y \\
& x_{t}\left(a_{0}, a_{1}, a_{2}, a_{3}, x, y\right)=a_{0}+a_{1} x+a_{2} y+a_{3} x y
\end{aligned}
$$

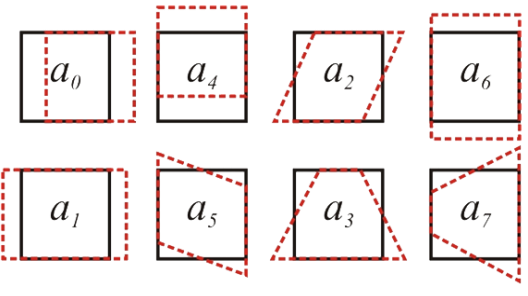

Figure 4

Transformation parameters

These parameters are determined by minimizing the distance between the observed grey pattern $G_{2}(x, y)$ in the second image and the original pattern $G_{1}(x, y)$ and by applying the photogrammetric corrections as follows [5]:

$\min _{a_{0}, \ldots a_{7}, g_{0}, g_{1}} \sum_{x, y}\left\|G_{1}(x, y)-G_{T}(x, y)\right\|$.

The term $G_{T}(x, y)$ represents an intensity change of grey points during loading and can be expressed:

$G_{T}(x, y)=g_{0}+g_{1} \cdot G_{2}\left(x_{t}(x, y), y_{t}(x, y)\right)$,

where $g_{0}$ and $g_{1}$ are the illumination parameters.

If the initial contour and displacement vectors of all element points are known, it is possible to compute its strains. Acquisition of strains is ensured either by differentiation of adjoining point displacements or by analysis of local facets curving used by correlation. 


\section{Background of Operational Modal Analysis}

The dynamic behavior of any mechanical system can be described in frequency domain by Frequency Response Functions (FRFs). Consider the model of MDOF system with $N$ degrees of freedom. Its FRFs can be written as a function of the complex poles as follows $[6,7]$ :

$\mathbf{H}(j \omega)=\sum_{i=1}^{N}\left(\frac{\mathbf{R}_{r}}{j \omega-\lambda_{r}}+\frac{\mathbf{R}_{r}^{*}}{j \omega-\lambda_{r}^{*}}\right)$,

where $\mathbf{H}$ is the matrix of Frequency Response Functions.

The poles are expressed by equation:

$\lambda_{r}=\sigma_{r}+j \omega_{r}$,

where $\sigma_{r}$ is the damping factor, and $\omega_{r}$ is the damped natural resonance frequency.

If the poles are considered as eigenvalues of a system, its eigenvectors can be interpreted as the modal shapes, where each eigenvector corresponds with a specific eigenvalue. The residues, $\mathbf{R}_{r}$ are described by equation $[6,7]$ :

$\mathbf{R}_{r}=\boldsymbol{\Psi}_{r} \boldsymbol{\gamma}_{r}^{T}$,

where $\gamma_{r}$ is the modal participation vector, $\boldsymbol{\Psi}_{r}$ is the mode shape. All those parameters are specified for the $r$-th mode.

It is obvious that FRFs depend on the modal parameters, and modal parameters can therefore be extracted from the FRFs.

Frequency response function matrix can also be expressed as the ratio between output (response) and input (excitation) as a function of frequency:

$\mathbf{H}(j \omega)=\frac{\mathbf{Y}(j \omega)}{\mathbf{X}(j \omega)}$.

This concept is commonly used in practical applications of Experimental Modal Analysis. Excitation is any form of input that is used to create a response in a structural system. In Experimental Modal Analysis, there is controlled force signal used as input. The most commonly-used excitation signals are harmonic or periodic signals, random signals, impact (impulse) etc.

Operational modal analysis allows obtain a modal model of the system by using only the measured response and without explicitly knowing the excitation input. The relationship between input and output (eq. 6) can be also written in the following form $[6,7]$ : 


$$
\mathbf{G}_{y y}(j \omega)=\mathbf{H}(j \omega)^{*} \mathbf{G}_{x x}(j \omega) \mathbf{H}(j \omega)^{T},
$$

where $\mathbf{G}_{x x}(j \omega)$ is the input spectral density matrix and $\mathbf{G}_{y y}(j \omega)$ is the output spectral density matrix. Since $\mathbf{G}_{x x}(j \omega)$ is not known in OMA, it is necessary to make an assumption that input is represented by a white noise that has a flat power spectral density over the entire frequency spectrum [8]. Then spectral density matrix of the measured responses can be expressed as $[6,7]$ :

$$
\mathbf{G}_{y y}(j \omega)=\sum_{r \in s u b(j \omega)}\left(\frac{d_{r} \boldsymbol{\Psi}_{r} \boldsymbol{\Psi}_{r}^{T}}{j \omega-\lambda_{r}}+\frac{d_{r}^{*} \boldsymbol{\Psi}_{r}^{*} \boldsymbol{\Psi}_{r}^{* T}}{j \omega-\lambda_{r}^{*}}\right),
$$

where $d_{r}$ is a scalar constant and $\operatorname{sub}(j \omega)$ is the set of modes that contribute at the particular frequency.

In a practical measurement, output spectral density matrix is defined as follows [6]:

$$
\mathbf{G}_{y y}(j \omega)=\left[\begin{array}{cccc}
g_{11}(j \omega) & g_{12}(j \omega) & \ldots & g_{1 \mathrm{n}}(j \omega) \\
g_{21}(j \omega) & g_{22}(j \omega) & \ldots & g_{2 \mathrm{n}}(j \omega) \\
\vdots & \vdots & \ddots & \vdots \\
g_{\mathrm{n} 1}(j \omega) & g_{\mathrm{n} 2}(j \omega) & \ldots & g_{\mathrm{nn}}(j \omega)
\end{array}\right]_{n \times n}
$$

where each element $g_{i j}$ is a spectral density function. The diagonal elements are the power spectral densities (PSD) of the corresponding response, the off-diagonal elements are the cross spectral densities (CSD) between the individual responses. Output spectral density matrix (for a certain frequency) is Hermitian $(n \times n)$, where $n$ being the number of measured responses.

The most important step of operational modal analysis is to extract modal parameters from the measurement data. Brincker et al. introduced method called Frequency Domain Decomposition (FDD) [9-11] that is based on the singular value decomposition of the output PSD matrix:

$$
\mathbf{G}_{y y}(j \omega)=\mathbf{U}(j \omega)^{*} \mathbf{S}(j \omega) \mathbf{V}(j \omega)^{H},
$$

where $\mathbf{U}(j \omega)$ and $\mathbf{V}(j \omega)$ are unitary matrices of singular vectors, and $\mathbf{S}(j \omega)$ is a diagonal matrix of singular values. In this special case, $\mathbf{U}(j \omega)$ and $\mathbf{V}(j \omega)$ are identical because $\mathbf{G}_{y y}(j \omega)$ is normal.

This technique allows us to identify possible coupled modes that are often indiscernible in the frequency spectrum. If only one mode is dominating at a particular frequency, then only one singular value will be dominating at this frequency and the corresponding singular vector is an estimate of the mode shape for that resonance frequency. Therefore, the first singular vector is a good 
approximation of the mode shape. In the case of close or repeated modes, there will be as many dominating singular values as there are close or repeated modes [6]. The plot of the first four or five singular values depending on frequency is sufficient to detect resonance peaks (Fig. 5). The frequency domain decomposition provides very good results for the resonance frequencies and the mode shapes. It is important to note that the obtained mode shapes are not true mode shapes because residues are not scaled to the input force.

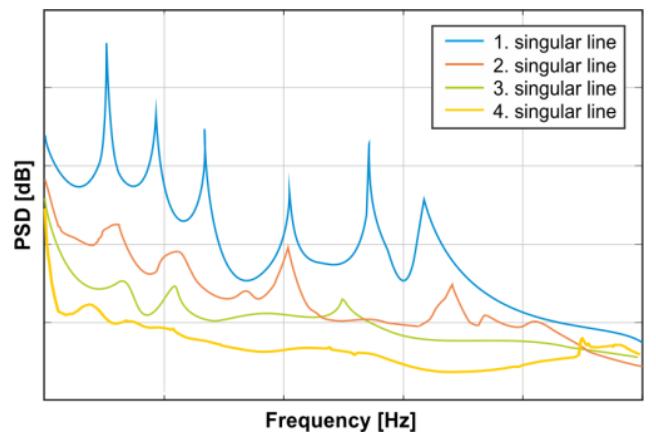

Figure 5

Plot example of singular values

It is also possible to obtain damping characteristics of each mode and more precise resonance frequencies by using the Enhanced Frequency Domain Decomposition (EFDD) based on the determination of the correlation functions [12]. The correlation function is being obtained from the power spectral density function by using the inverse Fast Fourier Transformation [13] and represents the free-decay of an equivalent SDOF oscillator. Fig. 6 shows an example of spectral Bell identification. This is the first step how to obtain a correlation function. Frequency band around the selected peak is defined by using Modal Assurance Criterion (eq. 13) of which value is greater than 0.9 [6-8]. Correlation function subsequently obtained by inverse FFT is shown in Fig. 7.

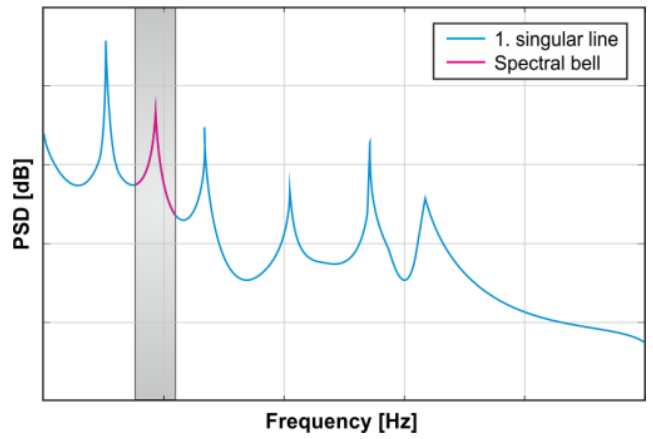

Figure 6

The identification of SDOF Bell function 


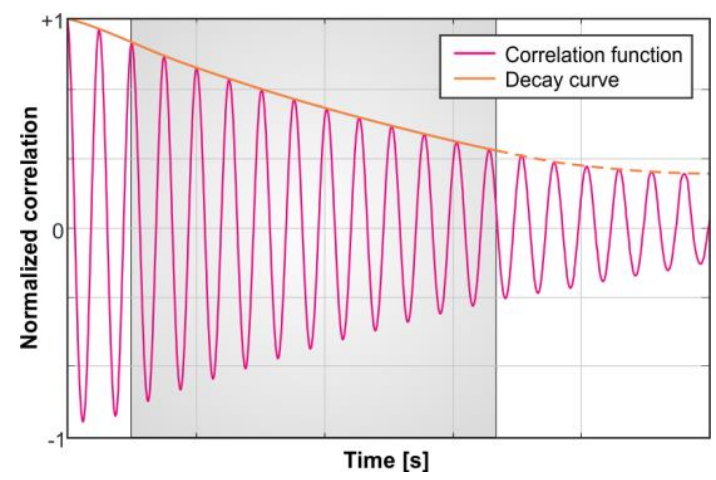

Figure 7

Correlation function of estimated SDOF Bell function

The resonance frequency is simply obtained by counting the number of times the correlation function crosses the zero axis. Damping is estimated by the logarithmic decrement technique and the logarithmic plot of decay curve [12] (Fig. 8). The logarithmic decrement $\delta$ is given by the equation [6, 7]:

$\delta=\frac{2}{n} \ln \left|\frac{r_{0}}{r_{n}}\right|$,

where $r_{0}$ is the initial value of the correlation function and $r_{k}$ is the $k$-th extreme. Thus, logarithmic decrement and the initial value of the correlation function can be found by linear regression on $k \delta$ and $2 \ln \left|r_{n}\right|$. The damping ratio for the mode $k$ is given by the well-known formula $[6,7]$ :

$\xi_{k}=\frac{\delta_{k}}{\sqrt{\delta_{k}^{2}+4 \pi^{2}}}$.

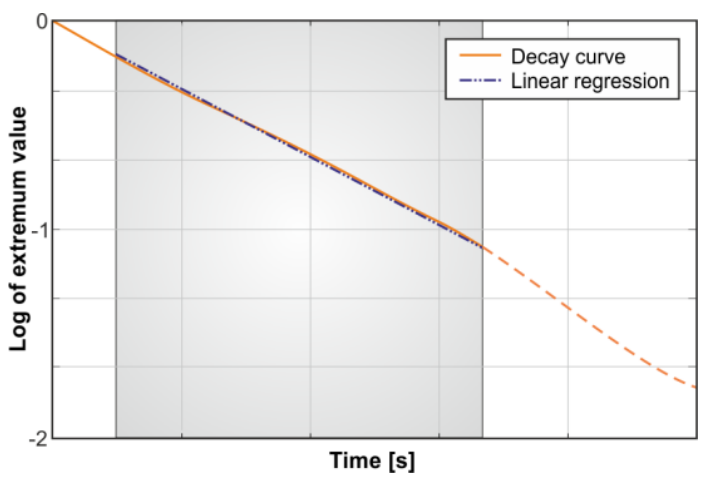

Figure 8

Estimation of damping ratio 
As Fig. 8 shows, only the part of the correlation function is used for the estimation, because they are dominated nonlinearities at the beginning and the end of correlation function.

The Modal Assurance Criterion (MAC) can be also used to investigate the validity of estimated modes. MAC is a mathematical tool to compare two vectors to each other. The MAC value between two mode shape vectors $\boldsymbol{\Psi}_{r}$ and $\boldsymbol{\Psi}_{s}$ is calculated as [14]:

$$
\mathrm{MAC}_{r, s}=\frac{\left|\boldsymbol{\Psi}_{r}^{H} \boldsymbol{\Psi}_{s}\right|^{2}}{\boldsymbol{\Psi}_{r}^{H} \boldsymbol{\Psi}_{r} \boldsymbol{\Psi}_{s}^{H} \boldsymbol{\Psi}_{s}} .
$$

The MAC takes the values from the interval $(0,1)$. If the MAC is equal to 1 , mode shape vectors $\boldsymbol{\Psi}_{r}$ and $\boldsymbol{\Psi}_{s}$ are the same mode shape. If they are different the MAC value should be low, due to the orthogonality condition of the mode shapes.

\section{Experimental Determination of Modal Parameters by using DIC Method}

The using of DIC method in Operational Modal Analysis is based on postprocessing of the measurement data. Since, most correlation systems do not have software tools for the evaluation of such type of analysis, it is needed to use another post-proccessing application. Modan 3D is a modal analysis software tool intended for the high-speed digital image correlation system Dantec Dynamics Q450. It is being developed at the Department of Applied Mechanics and Mechatronics, Faculty of Mechanical Engineering, Technical University of Košice, Slovakia $[1,15,16]$.

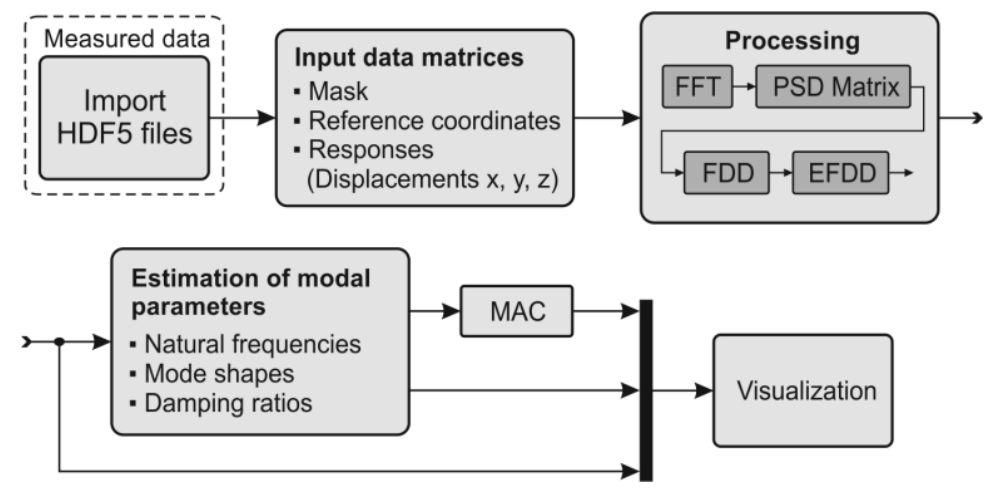

Figure 9

Principle scheme of Modan 3D software as a tool for operational modal analysis 
Modan is being programmed in Matlab because of easy transfer and processing of measurement data exported from Istra 4D (software application of the system Q450). Modan 3D has two main parts, of which one provides the evaluation for operational modal analysis measurements. This part uses functions and algorithms described in the previous chapter. The block diagram in Fig. 9 shows the basic principle of Modan 3D as a tool for operational modal analysis. Measured data exported from Istra4D are contained in the HDF5 files. The number of files corresponds to the number of time data. Each file contains the information about the surface mask and surface point displacements that represent measured responses. Modan enters these data into the relevant matrices. Subsequently, Power Spectral Density Matrix is being obtained by using Fast Fourier Transformation. PSD Matrix is important for the identification and estimation of modal parameters. Modan uses the algorithm of Frequency Domain Decomposition to determine natural frequencies and mode shapes. Damping ratio is being determined by Enhanced Frequency Domain Decomposition. The results of evaluation can be exported in graphical and text form.

The object of measurement was a steel specimen of fan blade shape with thickness of approximately $0.4 \mathrm{~mm}$. The specimen was fixed on the tapering side. For the purposes of image correlation, a black and white speckled pattern was sprayed onto the investigated surface. The specimen was acoustically excited by using the powerful sound system (Fig. 10) and white noise was used as the excitation signal. The measurement has been performed by using the correlation system Q-450 with two IDT NanoSense cameras.

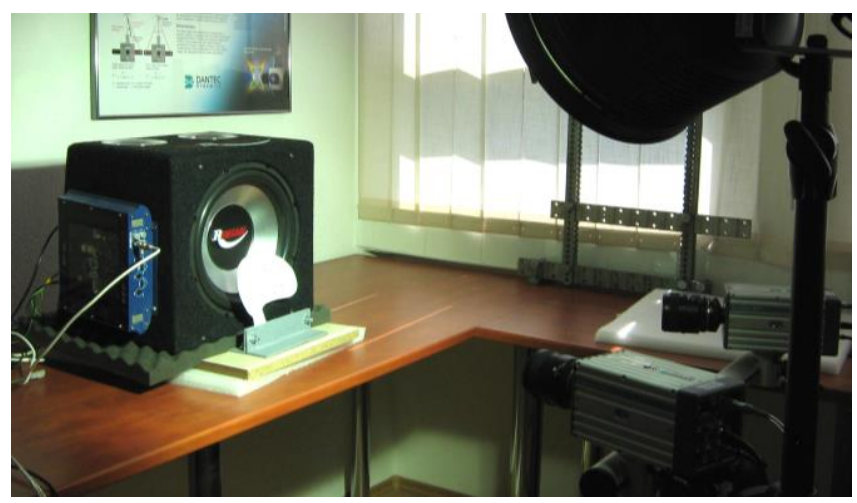

Figure 10

Experimental setup during the measurement

The sampling frequency was $1000 \mathrm{fps}$ and the total acquisition time was 1 second. Coordinates and spatial displacements of all surface points were determined in image correlation process in every single time step. The correlation results have been exported from Istra4D in HDF5 file format and subsequently processed in Modan 3D. 
The reference specimen shape shown in Fig. 11 is the result of the image correlation and contains 1831 mesh points. Computer memory size is the limiting factor in creating of spectral density matrices. When we used all points, the only one complete spectral density matrix had more than 1.3 billion elements. For this reason, Modan 3D had to reduce the number of the input data. The size of the matrices used in the experiment was $237 \times 237 \times 500$.

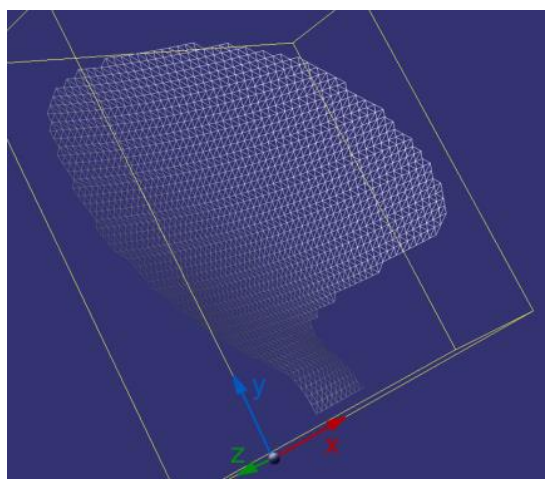

Figure 11

The reference shape of specimen

Fig. 12 shows the first four singular lines obtained by the singular value decomposition of the output spectral density matrix for vibration measured in $\mathrm{z}$ direction. Potential resonance frequencies were determined from the first singular line by peak-picking method. Their mode shapes vectors were subsequently compared to each other by using MAC (Fig. 13).

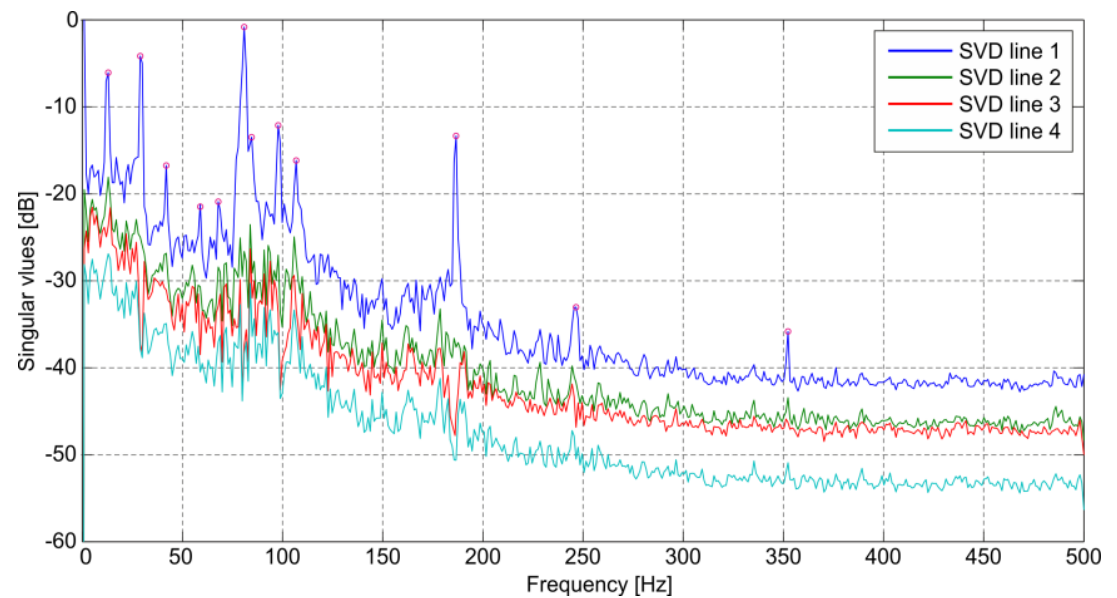

Figure 12

Singular lines for vibration measured in $\mathrm{z}$-direction 


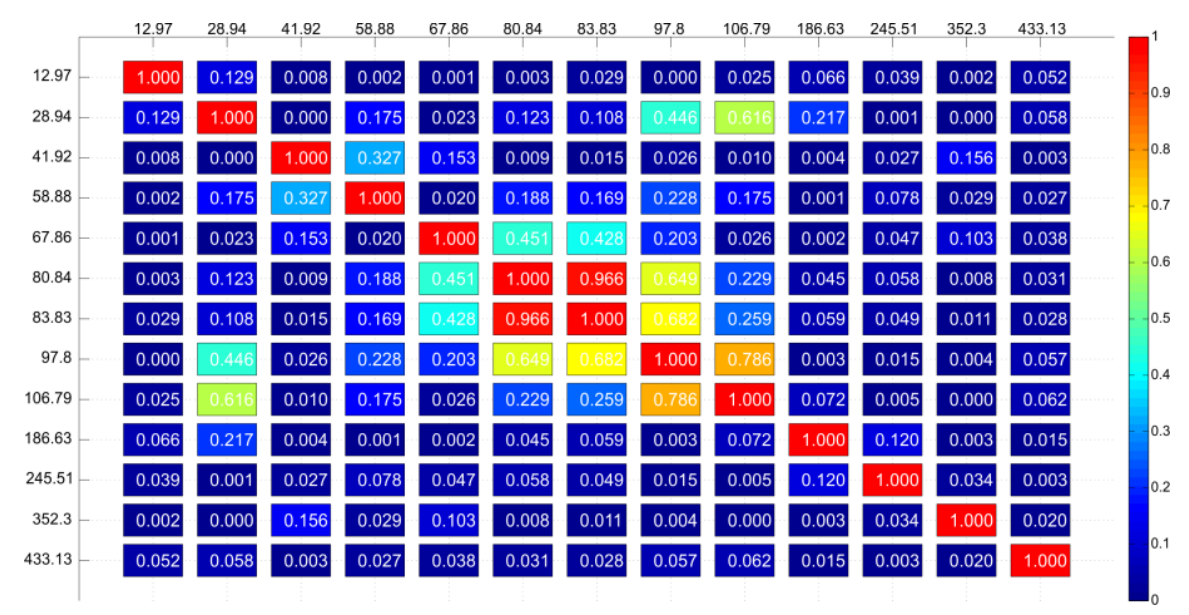

Figure 13

Auto MAC matrix

In frequency range up to $500 \mathrm{~Hz}$, there have been identified the first 7 modes. The individual resonance frequencies and their mode shapes obtained by Modan 3D are shown in Tab. 1.

The results of the modal analysis performed in the Modan 3D have been verified by using the finite element method (see Tab. 1). Istra4D allows to export obtained geometry to STL (STereoLithography) file format. Spatial contour of the specimen was imported to SolidWorks as an object of the type "surface". There were defined material properties and thickness of the imported surface. SHELL elements were used to meshing.

Table 1

Resonance frequencies and mode shapes of the specimen

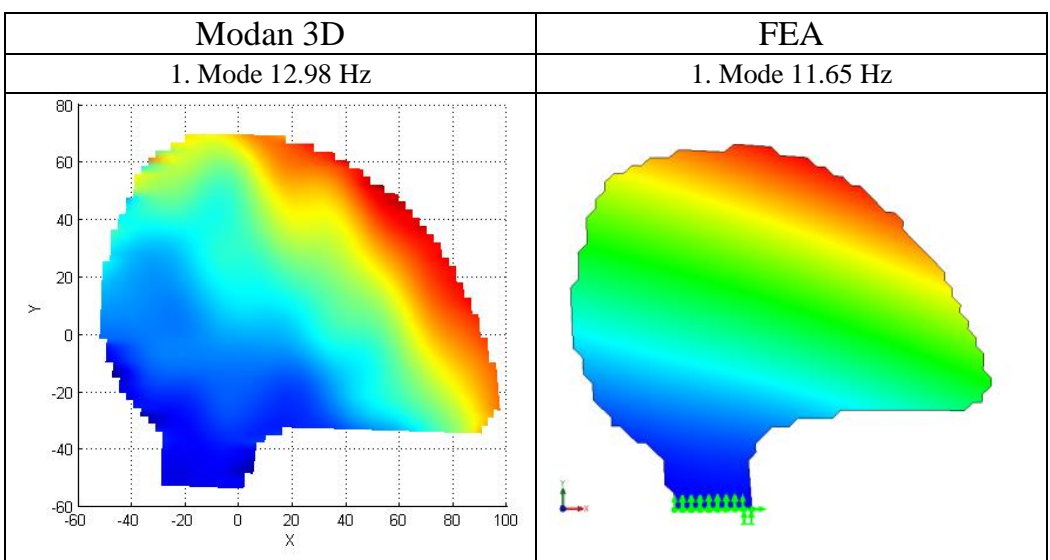


Table 1 (continue)

Resonance frequencies and mode shapes of the specimen

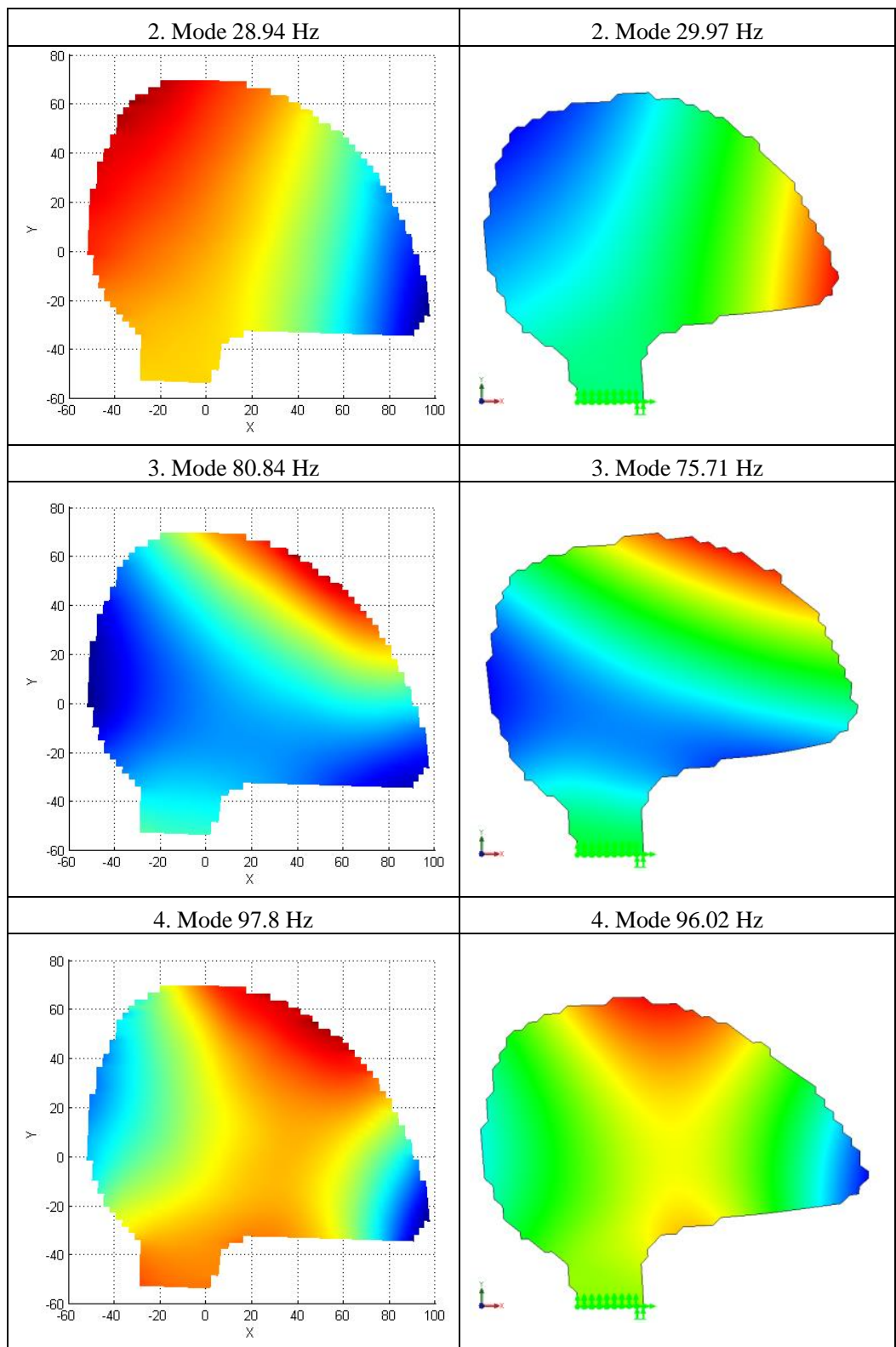


Table 1 (continue)

Resonance frequencies and mode shapes of the specimen

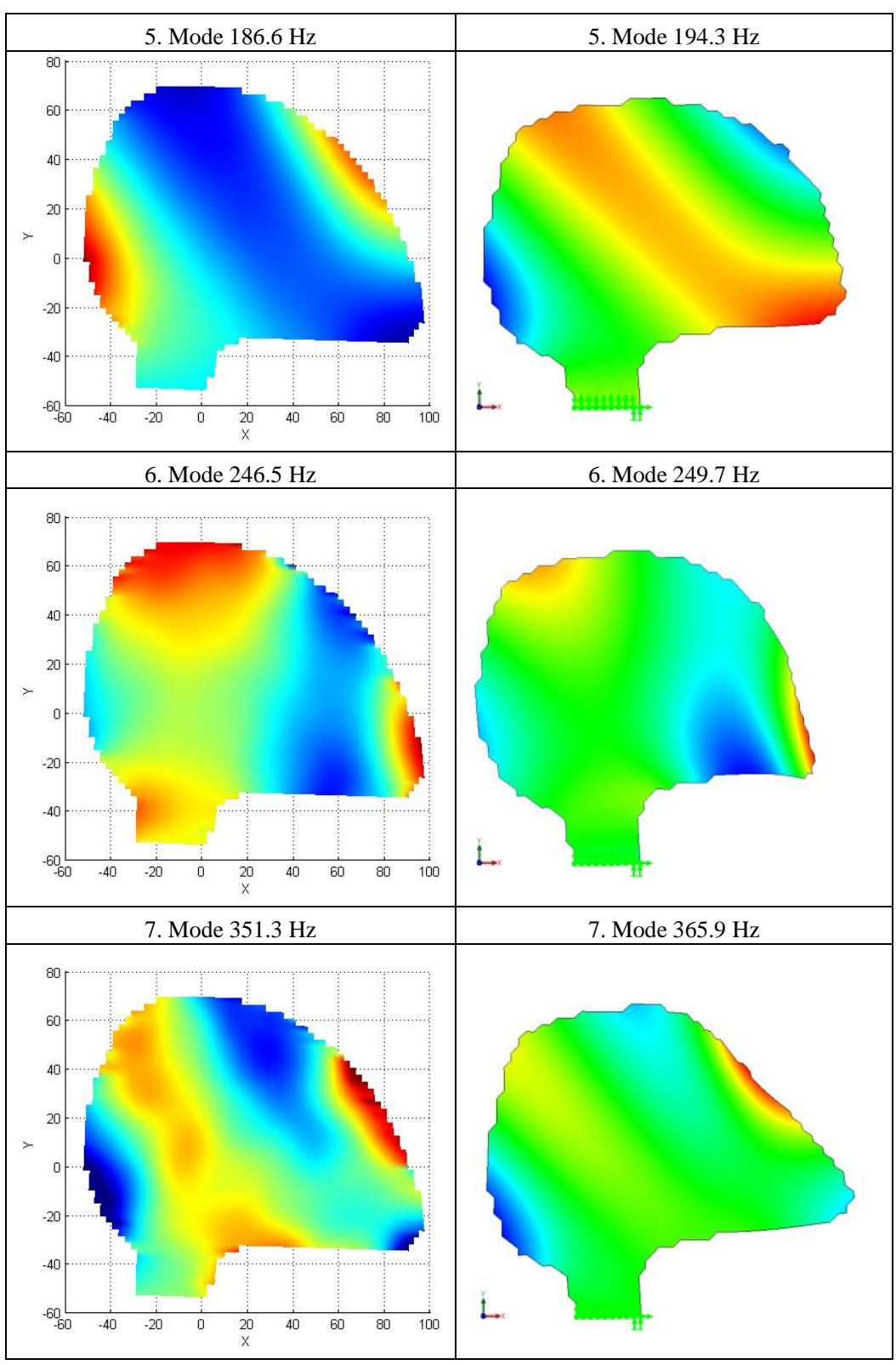


Modal parameters obtained by the measurement are in relatively good agreement with the results of numerical analysis. The differences in the mode shapes and frequency values may be caused by the fact that boundary conditions considered in the simulation are ideal - not real. In addition, the accuracy of the measurement depends on the measurement system sensitivity and on the parameters of FFT analyzer.

\section{Conclusions}

The flexible design of digital image correlation systems opens a wide range of their applications. Due to rapid new developments in high resolution digital cameras and computer technology, systems with high-speed cameras have proven to be a flexible and useful tool for vibration analysis and dynamic measurements. But, the full utilization of DIC method in Operational Modal Analysis or Experimental Modal Analysis requires the use of appropriate software application for the post-processing of the measurement data. In the paper, there was described a software tool called Modan 3D that is able to determine modal parameters by using the system Q-450. Modan 3D is actually not finished as well as some functions mentioned in the paper. There is needed to finalize algorithms for damping ratio estimation, perform complex verification of evaluation results and create a Graphical User Interface.

\section{Acknowledgement}

This work is supported by the projects VEGA 1/0937/12, VEGA 1/0289/11 and APVV-0091-11.

\section{References}

[1] M. Hagara, F. Trebuňa, R. Huňady, M. Kalina, M. Schrötter: Experimental Identification of Modal Parameters of Thin Metal Sheets by Using of DIC. In: Procedia Engineering 48, 2012, pp. 180-188

[2] M. Kalina, F. Šimčák, M. Hagara, M. Schrötter, M. Štamborská: The Use of the Experimental Optical Technique for Investigation of Shear Strains of the Samples Exposed to Shear Stress Beyond the Yield Point. In: Procedia Engineering 48, 2012, pp. 264-272

[3] C. Dudescu, A. Botean, M. Hardau: Application of Digital Image Correlation for Measuring E-Modulus of Wood Beams. In. Annals of DAAAM for $2009 \&$ Proceedings of the $20^{\text {th }}$ International DAAAM Symposium, Vol. 20, No. 1, Vienna, Austria, 2009

[4] T. Siebert, K. Splitthof, S. Stecklum, Ch. Herbst: New Features in Digital Image Correlation Techniques. In: $22^{\text {nd }}$ Danubia-Adria Symposium on Experimental Methods in Solid Mechanics, Parma, Monticelli Terme, 2005

[5] T. Siebert, T. Becker, K. Splitthof: Analysis of Advanced Materials under Load. In: SPIE - The International Society for Optical Engineering, November 2006, SPIE Newsroom <http://spie.org/x8594.xml> 
[6] M. Batel: Operational Modal Analysis - Another Way of Doing Modal Testing. In: Sound and Vibration, August, 2002, pp. 22-27 <http://www.sandv.com/downloads/0208batl.pdf>

[7] B. MacMillan, M. Batel, E. Dascotte, B. Verbeeck: OMA Testing by SLDV with FEM Pre- and Post-Test Analysis. In: Proceedings of the $22^{\text {th }}$ International Modal Analysis Conference (IMAC), January 2004, Detroit, Michigan

<http://www.femtools.com/download.php?id=imac2004b\&dl=no>

[8] C. Rainieri, G. Fabbrocino, G. Manfredi, M. Dolce: Robust Output-Only Modal Identification and Monitoring of Buildings in the presence of Dynamic Interactions for Rapid Post-Earthquake emergency Management. In: Engineering Structures 34, 2012, pp. 436-446

[9] R. Brincker, C. E. Ventura, P. Andersen: Damping Estimation by Frequency Domain Decomposition. In: Proceedings of IMAC 19: A Conference on Structural Dynamics, Hyatt Orlando, Kissimmee, Florida, 2001, Society for Experimental Mechanics, 2001. pp. 698-703

[10] R. Brincker, L. Zhang, P. Andersen: Modal Identification From Ambient Responses Using Frequency Domain Decomposition. In: Proceedings of The $18^{\text {th }}$ International Modal Analysis Conference (IMAC), San Antonio, Texas, 2000, pp. 625-630

[11] N. J. Jacobsen, P. Andersen, R. Brincker: Using EFDD as a Robust Technique to Deterministic Excitation in Operational Modal Analysis. In: Proceedings of The $2^{\text {nd }}$ International Operational Modal Analysis Conference (IOMAC), Copenhagen, Denmark, 2007

[12] F. Gomaa, M. Tayel, K. Kandil, G. Hekal: Validation Study Illustrates the Accuracy of Operational Modal Analysis Identification. In. International Journal of Emerging Technology and Advanced Engineering, Vol. 2, Issue 11, November 2012 <http://www.ijetae.com/files/Volume2Issue11/IJETAE_1112_103.pdf>

[13] E. Reynders: System Identification Methods for (Operational) Modal Analysis: Review and Comparison. In: Archives of Computational Methods in Engineering, Vol. 19, Issue 1, March 2012 pp. 51-124

[14] R. J. Allemang: The Modal Assurance Criterion - Twenty Years of Use and Abuse. In: Sound and Vibration, August, 2003, pp. 14-21

[15] R. Huňady, M. Hagara, M. Schrötter: Using High-Speed Digital Image Correlation to determine the Damping Ratio. In: Procedia Engineering 48, 2012, pp. 242-249

[16] F. Trebuňa, R. Huňady, Z. Bobovský, M. Hagara: An Application of Highspeed Digital Image Correlation in Determination of Modal Parameters. In: Acta Mechanica Slovaca, Vol. 15, No. 4, 2011, pp. 6-12 